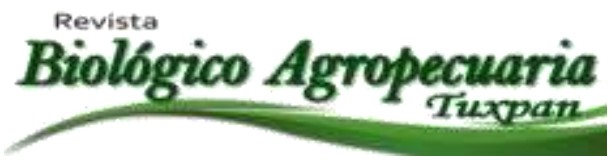

\title{
Indicadores de confort en vacas lecheras estabuladas en la región de tuxpan, veracruz y en el altiplano
}

Indicators of comfort in housed dairy cows in the region of tuxpan, veracruz and the highlands

$$
\begin{gathered}
\text { Lammoglia Miguel Ángel }^{1 凶}{ }^{\bowtie} \text { López-Castro Rubén Dario }^{1} \text {, Cabrera Amalia }{ }^{1} \text {, Daniel } \\
\text { Iliana C. }{ }^{1} \text { y Garcez Nora }{ }^{1}
\end{gathered}
$$

${ }^{1}$ Facultad de Ciencias Biológicas y Agropecuarias, Universidad Veracruzana, Poza Rica-Tuxpan Carretera Tuxpan-Tampico, km 7.5, Tuxpan, Ver. Tel y Fax: 017838344350.

${ }^{\square}$ Autor para correspondencia: malammoglia@hotmail.com

Recibido: 08/01/2014
Aceptado: 16/07/2014

\section{RESUMEN}

El objetivo del estudio fue determinar el confort de vacas lecheras especializadas y estabuladas en la región de Tuxpan, Veracruz y el Altiplano durante el mes de junio del 2013 utilizando diferentes indicadores (Parada=PA; Comiendo=C; Echada $=\mathrm{E}$; en ordeña=OR). Se utilizaron 2 establos uno en el altiplano con 3 diferentes tipos de corral: 1) nave moderna (NM), 2) nave moderna con nebulizadores (NMN) y 3) nave vieja (NV) y el otro en la región de Tuxpan, Veracruz con 2 diferentes tipos de corral 1) con mejor sombra (MS) y 2) sombra disminuida (SD). Se observaron 3 vacas por corral por 2 periodos de 24 horas. Se utilizó ANOVA para analizar los datos. No hubo diferencia $(\mathrm{P}=.10)$ entre los establos pero si un efecto del corral $(\mathrm{P}=.05)$ en los indicadores de confort. Las vacas pasaron más

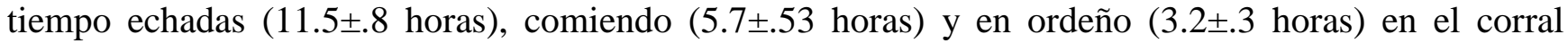

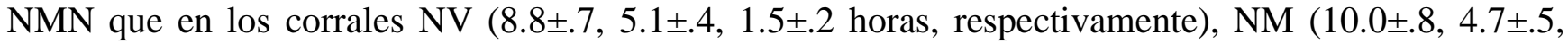

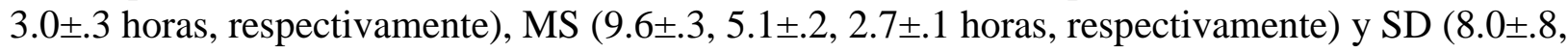

$4.9 \pm .5$, y $2.8 \pm .3$ horas, respectivamente). Las vacas pasaron más tiempo paradas en el corral NV que en los demás corrales. En conclusión, las condiciones del corral en ganado lechero estabulado pueden influenciar las horas de descanso y comida así como las horas que pasan paradas, y por lo tanto el confort de las vacas.

Palabras clave: confort, indicadores, echadas, corral.

\begin{abstract}
The aim of the study was to determine the comfort of housed dairy cows in the region of Tuxpan, Veracruz and the highlands during June 2013 using different indicators (standing = PA; Eating = C; lying down $=\mathrm{E}$, at milking $=\mathrm{OR}$ ). We used two farms, one in the highlands with 3 different types of
\end{abstract}


barns: 1) modern (NM), 2) modern with nebulizers (NMN) and 3) old (NV) and the other in the region of Tuxpan, Veracruz with 2 different types of barns 1) with best shade (MS) and 2) decreased shade (SD). Cows were observed ( 3 cows per barn) for 2 periods of 24 hours. ANOVA was used to analyze the data. There was no difference $(\mathrm{P}=.10)$ between the farms but there was a barn effect $(\mathrm{P}=.05)$ in the comfort indicators. The cows spent more time lying down (11.5 \pm 0.8 hours), eating $(5.7 \pm 0.53$ hours) and at milking (3.2 \pm 0.3 hours) in the barn NMN than in the barns $N V(8.8 \pm 0.7,5.1 \pm .4,1.5 \pm 0.2$ hours, respectively), NM (10.0 $\pm 0.8,4.7 \pm 0.5,3.0 \pm 0.3$ hours, respectively), MS $(9.6 \pm 0.3,5.1 \pm 0.2,2.7 \pm 0.1$ hours, respectively) and SD $(8.0 \pm 0.8,4.9 \pm 0.5$, and $2.8 \pm 0.3$ hours, respectively). The cows in the NV barn spent more time standing than in the other pens. In conclusion, barn conditions can influence the time cows spend resting, eating and standing and therefore cow's comfort.

Keywords: comfort, indicators, housed cows,

\section{INTRODUCCIÓN}

El bienestar del ganado bovino se encuentra influenciado por factores como la temperatura y humedad relativa, las instalaciones, el manejo zootécnico, la nutrición y la medicina preventiva, principalmente (Gary, 1987). El comportamiento de los animales es un indicativo de la relación de éstos con el sistema de producción en el cual viven; por tanto, su identificación puede representar un punto de partida para evaluar el bienestar (Duncan, 1990). Los bovinos mantenidos y manejados en condiciones de bienestar pueden expresar mejor sus características genéticas, lo cual favorece económicamente la producción (Árabe y Albright, 1997).

Los cambios en los patrones de comportamiento animal del ganado vacuno de leche son ampliamente utilizados como una medida de bienestar animal. La duración y frecuencia con la que determinadas actividades de comportamiento (echada, de pie, comiendo/pastoreando y rumiando) tienen lugar al mismo tiempo dentro del rebaño, tanto en pastoreo como en establo, nos da una idea del grado de sincronía de comportamientos y del estado de bienestar del rebaño lechero (Miller y Wood-Gush, 1991). Es importante que en los sistemas de manejo no se dificulte o impida que los animales tengan un descanso adecuado (Drissler et al. 2005). Overton et al. (2002) señalaron además que la mayor parte del tiempo que una vaca no está comiendo debe estar echada y produciendo leche.

La actividad de pastar cuando no se limita el tiempo de pastoreo, se encuentra muy influenciada por el número de horas de luz (Linnane et al., 2001), incluso el regreso del ordeño estimula el deseo de comer (O'Connell et al. 1989). Esto sucede también en las vacas estabuladas, en las cuales la actividad de comer se ve altamente potencializada por el reparto de silo fresco (DeVries et al. 2005). Diferentes autores (Fisher et al. 2003; Drissler et al. 2005) evaluaron el confort de diferentes sistemas de producción en función del número de horas diarias que las vacas permanecen echadas y esto, a su vez, lo relacionaron con la calidad del área de reposo (Manninen et al. 2002) manifestando la importancia de una cama cómoda y de dimensiones apropiadas para facilitar el descanso en vacas estabuladas. El objetivo del estudio fue determinar el confort de vacas lecheras especializadas y estabuladas en la región de Tuxpan, Veracruz y el Altiplano durante el mes de junio del 2013 utilizando diferentes indicadores.

Revista Científica Biológico Agropecuaria Tuxpan 2 (1) 


\section{MATERIALES Y MÉTODOS}

El presente estudio de investigación se realizó en 2 ranchos: 1) Rancho Santa Clara localizado en la zona norte del estado de Veracruz en la carretera Alamo-Alazán km 56 en el municipio de Tepetzintla y se observó el comportamiento de las vacas en 2 diferentes tipos de corral: 1) con mejor sombra (MS) y 2) sombra disminuida (SD) y 2) rancho La Quinta ubicado en el km 7, carretera ActopanChicavasco, municipio de Actopan, Hidalgo. En el rancho La Quinta se observó el comportamiento de las vacas en 3 diferentes tipos de corral: 1) nave moderna (NM), 2) nave moderna con nebulizadores (NMN) y 3) nave vieja $(\mathrm{NV})$.

En los ranchos Santa Clara y la Quinta se eligieron aleatoriamente 3 vacas primíparas y 3 vacas multíparas por corral y se observó y anotó detalladamente las actividades realizadas de estas vacas durante periodos de 24 horas continuas. La edad de la vaca y la etapa de lactancia se determinaron de acuerdo con los registros individuales existentes en los ranchos. Durante el mes de junio del 2013 se realizaron 3 réplicas de observación continua durante periodos de 24 horas de las conductas individuales y sociales de cada una de las vacas. Las actividades observadas fueron las siguientes: tiempo dedicado a estar parada, comiendo, caminando, echada, echada rumiando, tomando agua, parada rumiando, $\mathrm{y}$ ordeñando.

El análisis estadístico se realizó utilizando un programa de análisis de varianza (ANOVA) del software Statistica 7.

\section{RESULTADOS}

No hubo diferencia $(\mathrm{P}=.10)$ del tiempo dedicado a las diferentes actividades afectadas por el rancho pero si un efecto del corral $(\mathrm{P}=.05)$ en los indicadores de confort (Tabla 1).

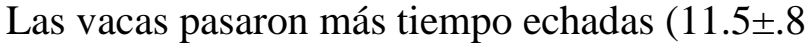
horas), comiendo (5.7 \pm .53 horas) y en ordeño (3.2 \pm .3 horas) en el corral NMN (Cuadro 1) que en los corrales $\mathrm{NV}(8.8 \pm .7,5.1 \pm .4,1.5 \pm .2$ horas, respectivamente), NM (10.0 $\pm .8,4.7 \pm .5$, $3.0 \pm .3$ horas, respectivamente), MS (9.6 \pm .3 , $5.1 \pm .2,2.7 \pm .1$ horas, respectivamente) y $\mathrm{SD}$ $(8.0 \pm .8, \quad 4.9 \pm .5, \quad$ y $2.8 \pm .3$ horas, respectivamente). Las vacas pasaron menos tiempo caminando en el corral NMN que en los demás corrales (Tabla 1). Las vacas en los corrales viejos de la Quinta y las vacas del rancho Santa Clara con sombra disminuida pasaron más $(\mathrm{P}=.05)$ tiempo paradas que en el resto de los corrales (Tabla 1).

\section{DISCUSIÓN}

De acuerdo a los resultados obtenidos de este estudio las vacas en el corral de nave moderna y con nebulizadores del rancho la Quinta en el mes de junio del 2013 proporcionó a las vacas lecheras el mayor confort. Las vacas pasaron mayor tiempo descansando, comiendo y siendo ordeñadas. Esto también nos indicaría que las vacas en este corral deberían estar produciendo mas leche. Grant (2006) publicó que las vacas lecheras producían leche durante 3 situaciones: siendo ordeñadas, comiendo y en descanso y estas 3 categorías fueron alcanzadas en mayor tiempo en las vacas en el corral nuevo con nebulizadores del rancho la Quinta. Así mismo Grant (2006) reportó que por cada hora adicional de descanso al día la vaca puede producir $1 \mathrm{~kg}$ de leche más por día.

\section{Revista Científica Biológico Agropecuaria Tuxpan 2 (1) ISSN: 2007-6940}


Cuadro 1. Indicadores de confort en horas afectados $(\mathrm{P}=.05)$ por el tipo de corral en vacas lecheras estabuladas (Filas con diferente letra en superíndice difieren $\mathrm{P}=.05$ ).

\begin{tabular}{|c|c|c|c|c|c|}
\hline $\begin{array}{l}\text { INDICADORES } \\
\text { (en horas) }\end{array}$ & $\begin{array}{c}\text { La Quinta } \\
\text { Nave } \\
\text { Vieja }\end{array}$ & $\begin{array}{c}\text { La Quinta } \\
\text { Nave } \\
\text { Moderna }\end{array}$ & $\begin{array}{c}\text { La Quinta } \\
\text { Nave } \\
\text { Moderna con } \\
\text { nebulizador }\end{array}$ & $\begin{array}{c}\text { Santa Clara } \\
\text { Nave con } \\
\text { mayor } \\
\text { sombra }\end{array}$ & $\begin{array}{c}\text { Santa Clara } \\
\text { Nave con } \\
\text { sombra } \\
\text { disminuida }\end{array}$ \\
\hline Parada & $\begin{array}{c}4.80 \pm \\
0.65\end{array}$ & $3.28 \pm 0.81$ & $4.11 \pm 0.81$ & $4.00 \pm 0.30$ & $3.82 \pm 0.81$ \\
\hline Comiendo & $\begin{array}{c}5.06 \pm \\
0.43\end{array}$ & $4.66 \pm 0.53$ & $5.68 \pm 0.53$ & $5.09 \pm 0.21$ & $4.95 \pm 0.53$ \\
\hline Caminando & $\begin{array}{c}0.27 \pm \\
0.05^{\mathrm{b}}\end{array}$ & $0.70 \pm 0.06^{b}$ & $0.01 \pm 0.06^{\mathrm{a}}$ & $0.42 \pm 0.02^{b}$ & $0.72 \pm 0.06^{\mathrm{b}}$ \\
\hline Echada & $\begin{array}{c}6.49 \pm \\
0.47\end{array}$ & $8.80 \pm 0.58$ & $8.38 \pm 0.58$ & $7.40 \pm 0.22$ & $5.95 \pm 0.58$ \\
\hline Echada rumiando & $\begin{array}{c}2.42 \pm \\
0.57\end{array}$ & $1.24 \pm 0.71$ & $2.63 \pm 0.71$ & $2.07 \pm 0.26$ & $1.97 \pm 0.71$ \\
\hline Total descanso & $\begin{array}{c}8.82 \pm \\
0.67^{\mathrm{a}}\end{array}$ & $9.96 \pm 0.82^{\mathrm{a}}$ & $11.47 \pm 0.82^{b}$ & $9.57 \pm 0.31^{\mathrm{a}}$ & $8.02 \pm 0.82^{\mathrm{a}}$ \\
\hline Agua & $\begin{array}{c}0.28 \pm \\
0.06\end{array}$ & $0.33 \pm 0.07$ & $0.31 \pm 7.86$ & $0.36 \pm 0.03$ & $0.51 \pm 0.07$ \\
\hline Parada rumiando & $\begin{array}{c}2.44 \pm \\
0.58\end{array}$ & $1.49 \pm 0.71$ & $0.71 \pm .12$ & $1.52 \pm 0.27$ & $2.18 \pm 0.71$ \\
\hline Ordeño & $\begin{array}{l}1.54 \pm \\
0.23\end{array}$ & $2.97 \pm 0.29$ & $3.25 \pm 0.29$ & $2.66 \pm 0.11$ & $2.87 \pm 0.29$ \\
\hline Total parada & $\begin{array}{c}7.25 \pm \\
0.70^{\mathrm{a}}\end{array}$ & $4.77 \pm 0.62^{b}$ & $4.82 \pm 0.55^{b}$ & $5.52 \pm 0.73^{\mathrm{ab}}$ & $6.00 \pm 0.66^{\mathrm{a}}$ \\
\hline
\end{tabular}

Las vacas con mayor producción pasan al día alrededor de 12 a 14 horas descansando, 5.5 horas comiendo y de 2.5 a 3.5 horas ordeñando (Grant, 2006) que es similar a lo encontrado en las vacas de este estudio ubicadas en el corral moderno con nebulizadores del rancho la Quinta.

La diferencia con los otros corrales en los diferentes indicadores pueden haber sido afectados por sobrepoblación, áreas de descanso, disponibilidad de sombra, afectando las horas de descanso, comiendo y ordeñando (Callejo Ramos, 2009).

\section{CONCLUSIONES}

Las condiciones del corral en ganado lechero estabulado pueden influenciar las horas de descanso y comida así como las horas que 
pasan paradas, y por lo tanto el confort de las vacas.

\section{LITERATURA CITADA}

Árabe, C. W., y Albright, J. 1997. The L. Behavior of Cattle. $1^{\mathrm{a}}$ University ed., Press, Cambridge. UK.

Callejo-Ramos, A. 2009. Cow Comfort, El bienestar de la vaca lechera. Alojamiento y bienestar. pp. 141-142.

Duncan, J. H. 1990. Behavioral assessment of welfare. En: Mench, JA, SJ Mayer, andLKrulisch.(eds) TheWellBeing of A gricultural Animals in Biomedical and Agricultural Research. Scientists Center for Animal Welfare. Bethesda, Maryland. USA.
Grant, R. J. 2006. Incorporating dairy cow behavior into management tools. Proceedings from Penn State Dairy Cattle Nutrition Workshop.

Gary, P. M. 1987. A model for assessing the impact of behavioral stress on domestic animals. J Anim. Sci., 65: 1228-1235. Copyright (c) 2014 Miguel Angel Lammoglia Villagomez, Rubén López Castro, Amalia Cabrera Núñez, Iliana Del Carmen Daniel Renteria
y Nora Garcez

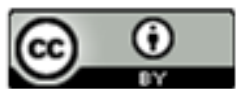

Este texto está protegido por una licencia Creative Commorr 4.0

Usted es libre para Compartir —copiar y redistribuir el material en cualquier medio o formato- y Ad aptar el documento —remezclar, transformar y crear a partir del material- para cualquier propósito, inchso para fines comerciales, siempre que cumpla la condición de:

Atribución: Usted debe dar crédito a la obra original de manera adecuada, proporcionar un enlace a la licencia, e indicar si se han realizado cambios. Puede hacerlo en cualquier forma razonable, pero no de forma tal que sugiera que tiene el apoyo del licenciante olo recibe por el usoque hace de la obra.

Resumende licencia - Textocompletodelalicencia 